\title{
Prise en compte des contraintes résiduelles de traitement thermique dans la prédiction de la tenue en service des culasses en aluminium
}

\author{
François Comte $^{1,2,3}$, T. Mac Lan Nguyen-Tajan ${ }^{1, a}$, Nicolas Morin $^{1}$, \\ Habibou Maitournam ${ }^{2}$ et Ziad Moumni ${ }^{3}$ \\ 1 PSA Peugeot Citroën, Direction de la Recherche et de l'Innovation, route de Gisy, 78943 Vélizy Villacoublay, France \\ 2 Laboratoire de Mécanique des Solides, CNRS UMR 7649, Dpt. de Mécanique, École polytechnique, 91128 Palaiseau, France \\ 3 UME/ENSTA, Chemin de la Hunière, 91761 Palaiseau, France
}

Reçu le 25 février 2005, accepté le 21 mars 2005

\begin{abstract}
Résumé - Cet article porte sur la modélisation des culasses aluminium de moteurs diesel HDI en vue de prédire leur tenue en fatigue polycyclique. Celles-ci, avant d'être frettées, serrées sur le bloc moteur et utilisées en service, subissent un traitement thermique (trempe et revenu) afin d'améliorer leurs propriétés mécaniques. Lors de ce procédé d'importantes contraintes résiduelles sont générées. Leur modélisation est nécessaire pour obtenir des résultats numériques prédictifs. Une première difficulté vient de la modélisation des échanges thermiques. Pour cela, le choix d'une fonction analytique simple pour le coefficient d'échange $H=f\left(T_{\text {paroi }}\right)$, suivi de l'optimisation des différents paramètres, a permis de modéliser les courbes de refroidissement. Une fois l'histoire thermique de la culasse modélisée, un calcul mécanique élastoplastique permet de déterminer les contraintes résiduelles. Celles-ci, fortement de traction dans la zone à risque, sont défavorables à la tenue à la fatigue polycyclique de la pièce dans le cas de la trempe à l'eau. La prise en compte de l'état résiduel de fin de traitement thermique dans la filière de dimensionnement à la fatigue polycyclique permet alors de caractériser les zones à risque expérimentalement observées.
\end{abstract}

Mots clés : Contraintes résiduelles / trempe / aluminium / fatigue polycyclique / modélisation numérique / culasse

\begin{abstract}
Thermal treatment residual stresses and high cycle fatigue of an aluminum alloy cylinder head. This paper deals with the computational modeling of the high cycle fatigue of the aluminum cylinder head (HDI diesel engine). Before being shrinked on, fixed onto the engine block and used, these components are submitted to a thermal treatment (quenching and ageing) to improve their mechanical properties. This gives rise to large residual stresses during quenching and therefore its modeling becomes necessary in order to obtain accurate numerical prediction. A series of tests was carried out at the PSA Peugeot Citroën production line from which the experimental cooling curves were determined. The experimental data were fitted with a simple analytical function $H=f\left(T_{\text {wall }}\right)$. Then, once the parameter identification was achieved, the remaining residual stresses followed from a simple mechanical analysis. It was found that the stresses in the local cracking failure area are tensile and therefore strongly unfavorable for the high-cycle fatigue endurance in the case of water quenching. In addition, the account of the residual stresses in the fatigue dimensioning, at the end of the thermal treatment, leads to predictions for the risk regions which are consistent with the experimental observations.
\end{abstract}

Key words: Residual stresses / quenching / aluminum / high cycle fatigue / numerical modeling / cylinder head

a Auteur correspondant : maclan.nguyen@mpsa.com 


\section{Introduction}

Dans le secteur automobile, du fait d'exigences de plus en plus sévères en terme de consommation, de dépollution, de performance, de coût et de fiabilité, les architectures et les solutions technologiques moteur sont amenées à évoluer. Leur conception repose notamment sur la modélisation numérique. De plus, de par la volonté de réduction des délais de conception et des coûts de développement, on doit passer d'un schéma de développement à grand nombre de prototypes physiques testés à un nouveau où la plus grande partie seront des prototypes virtuels. Par conséquent, afin de pouvoir appréhender les meilleures solutions envisageables, la simulation numérique est aujourd'hui devenue incontournable.

Par ailleurs la proportion des alliages légers, en particulier ceux en aluminium, est de plus en plus importante. C'est particulièrement vrai dans le cas des culasses. Ces dernières, coulées en fonderie, subissent ensuite un traitement thermique qui permet d'obtenir les propriétés métallurgiques optimales du matériau. Ce traitement thermique génère des contraintes résiduelles [1].

Les calculs de dimensionnement de la zone froide des culasses effectués aujourd'hui, sans prise en compte des contraintes issues du procédé de fabrication, ne permettent pas de prédire la fissuration expérimentale. L'introduction d'un état précontraint est nécessaire pour assurer une modélisation numérique prédictive et fiable de leur tenue à la fatigue polycyclique.

\section{Simulation du process de traitement thermique}

\subsection{Le process de traitement thermique}

Le traitement thermique subi par les culasses en aluminium se décompose en différentes étapes (Fig. 1) :

- une mise en solution pendant au moins 5 h à une température d'environ $540{ }^{\circ} \mathrm{C}$;

- une trempe à l'eau avec une température de bain d'environ $70{ }^{\circ} \mathrm{C}$;

- un revenu à $200{ }^{\circ} \mathrm{C}$ pendant $5 \mathrm{~h}$;

- un refroidissement à l'air ambiant.

Durant la première étape, le chauffage, dont la durée doit être suffisante et la température aussi proche que possible de la température de fusion, permet aux précipités de l'alliage d'entrer en solution solide. Cette étape n'est pas modélisée dans notre analyse; nous faisons l'hypothèse que la culasse est vierge de contraintes à la fin de la mise en solution.

La trempe fige ensuite la microstructure dans un état métastable donné. Le refroidissement violent à la température ambiante permet de maintenir, désormais en état de sursaturation, la solution solide existante.

Le revenu permet enfin l'obtention d'une multitude de petits précipités très fins à l'origine du durcissement

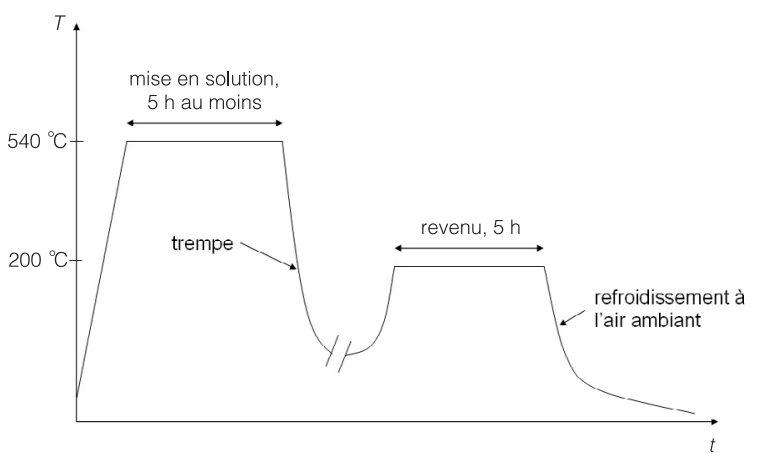

Fig. 1. Évolution de la température pendant le traitement thermique des culasses diesel aluminium.

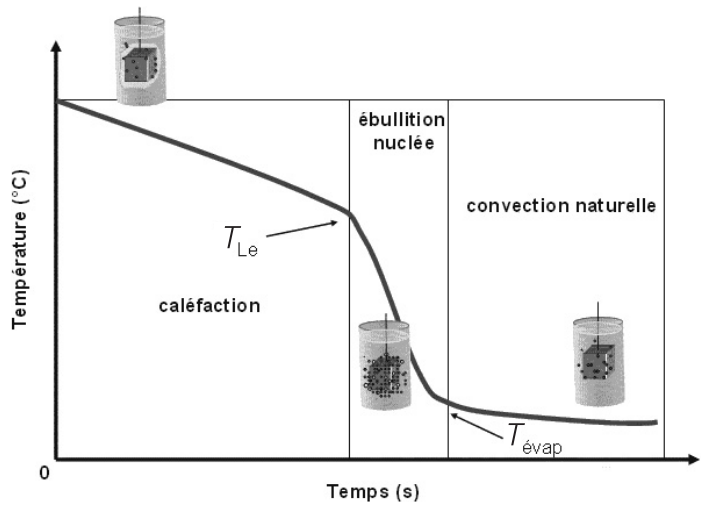

Fig. 2. Les trois étapes théoriques du refroidissement lors d'une trempe.

structural. Ceux-ci sont le résultat de la décomposition de la solution solide sursaturée de fin de trempe. La présence de ces petits précipités, qui s'opposent aux mouvements des dislocations, est à l'origine de l'amélioration des propriétés mécaniques.

\subsection{Identification de la thermique de trempe}

Au cours du processus de trempe ce sont les échanges thermiques entre les fluides vaporisables du milieu de trempe et la pièce qui pilotent la cinétique de refroidissement de la culasse. La vitesse de refroidissement n'est pas constante et trois grandes phases d'extraction de chaleur sont successivement observées [2] (Fig. 2).

La première étape est celle d'un refroidissement en phase vapeur appelée caléfaction. Elle se caractérise par la présence d'un film stable de vapeur autour de la totalité de la pièce dû à des températures de surface suffisamment hautes. À cause de la haute résistance thermique de la vapeur, le flux de chaleur extrait, principalement par conduction à travers ce film, est lent.

Pendant la deuxième étape, il apparaît des zones de contact direct entre la pièce et le liquide de trempe car l'évaporation n'est plus suffisante pour former une couche de vapeur continue. Il y a alors l'apparition de courants de convection et une ébullition nucléée, qui correspond à la phase d'extraction maximale de chaleur, apparaît. 


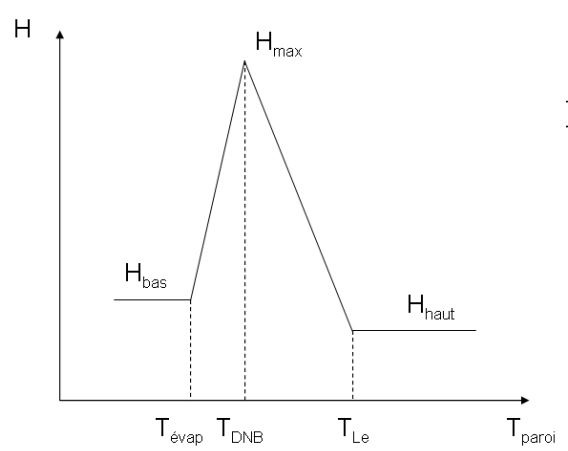

(a)

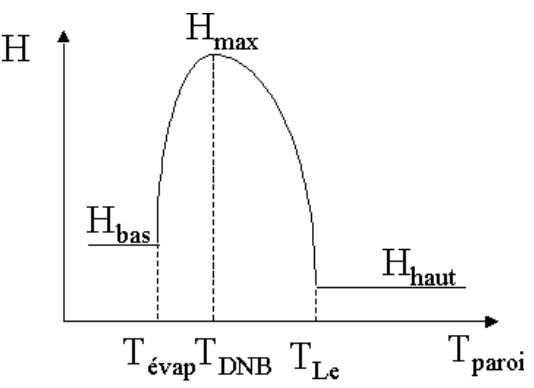

(b)

Fig. 3. Modélisation du coefficient d'échange sous forme de segments de droite (a) ou d'arcs de paraboles (b).

La troisième étape débute lorsque la température d'ébullition est atteinte à la surface de la pièce. Cette dernière est alors mouillée de manière définitive par le fluide et les échanges, relativement faibles, ne se font alors plus que par convection.

La température de transition entre les deux premières étapes s'appelle la température de Leidenfrost $T_{\mathrm{Le}}$.

Afin de prédire par le calcul le niveau des contraintes résiduelles, il est important de reproduire fidèlement l'histoire thermique de la culasse en modélisant correctement les échanges thermiques entre la culasse et le bain de trempe. À cette fin des mesures de température ont été effectuées à PSA sur la chaîne série de production des culasses aluminium. Plusieurs campagnes d'essais ont été réalisées et une bonne répétitivité du phénomène a pu être observée. La température de la pièce étant très inhomogène, plusieurs thermocouples ont été implémentés sur les différentes faces à quelques millimètres de la surface libre. L'objectif est alors de déterminer les coefficients d'échanges thermiques qui permettent de modéliser le refroidissement de la culasse et de vérifier que l'on retrouve bien par le calcul les valeurs relevées aux thermocouples.

Classiquement un coefficient d'échange unique $H(T)$ est déterminé pour toutes les surfaces en contact avec le liquide de trempe et l'on cherche à déterminer, pour toute une série de températures $\left\{T_{i}\right\}_{i \in\{1, n\}}$, les valeurs $H\left(T_{i}\right)[3,4]$. Dans notre cas, les faces ayant des géométries et des orientations dans le bain de trempe différentes, un coefficient d'échange différent sera déterminé pour chaque face. Dans ce cas, le nombre de valeurs $H\left(T_{i}\right)$ à déterminer devient très important si l'on désire que, pour chaque face, $H$ soit déterminé avec une discrétisation suffisamment fine en température. Le problème devient alors extrêmement complexe. Afin de le simplifier le choix d'une fonction analytique $H\left(T_{\text {paroi }}\right)$ permet de limiter le nombre de paramètres à identifier. Ehlers [5] et Jeschar [6] ont proposé d'approximer $H(T)$ par deux segments de droite pour le refroidissement en phase d'ébullition nucléée (Fig. 3a). Nous proposons d'approximer $H(T)$ par 2 arcs de parabole prolongés par des valeurs de $H$ constantes pour des températures $T>T_{\mathrm{Le}}$ et $T<T_{\text {évap }}$ (Fig. 3b). Cette modélisation permet en effet de mieux prendre en compte l'influence du paramètre

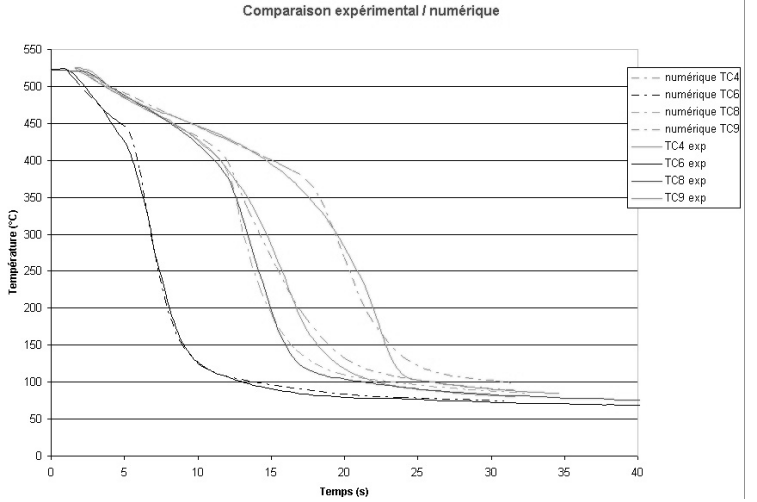

Fig. 4. Courbes de refroidissement (expérimentales et simulées) en divers points de la culasse.

$H_{\text {max }}$. Après optimisation (non détaillée dans cet article) des différents paramètres définissant les coefficients d'échange des différentes faces de la culasse, on trouve des courbes de refroidissement très comparables aux résultats expérimentaux (Fig. 4).

\subsection{Calcul mécanique}

C'est le refroidissement inhomogène, combiné à la rigidité de la pièce, qui est à l'origine des contraintes résiduelles. Pour les déterminer, on simule l'histoire thermique transitoire inhomogène de la culasse et on modélise le matériau avec différentes lois élastoplastiques à écrouissage cinématique linéaire en fonction des différentes étapes du traitement thermique. Ainsi une première loi est utilisée lors de l'étape de trempe et une seconde, avec des propriétés mécaniques supérieures, l'est à partir de la fin du revenu.

La figure 5 présente l'évolution de la pression hydrostatique $p$ lors de la trempe en deux points de la culasse : un premier sur l'extérieur de la pièce et un second à l'intérieur. On peut clairement observer, au cours du temps, une forte évolution des contraintes. L'intérieur en compression et l'extérieur en traction lors de la première partie du refroidissement passent respectivement en traction et compression à la fin du refroidissement. 


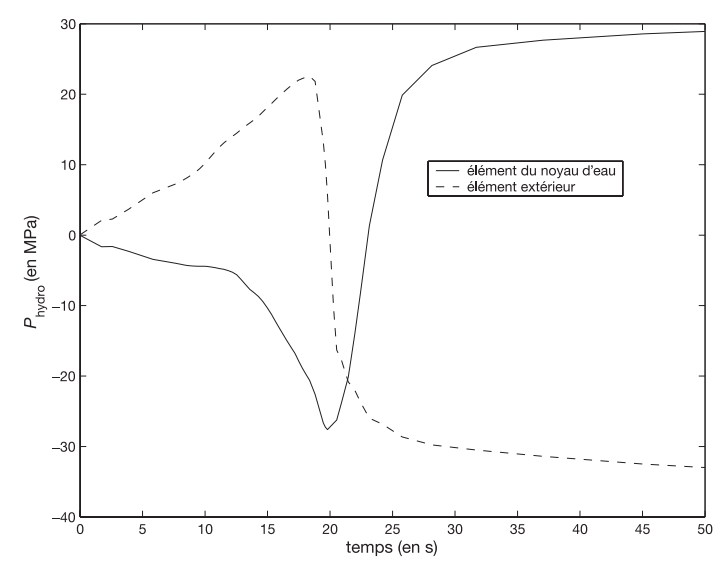

Fig. 5. Évolution de la pression hydrostatique lors de la trempe à l'eau.

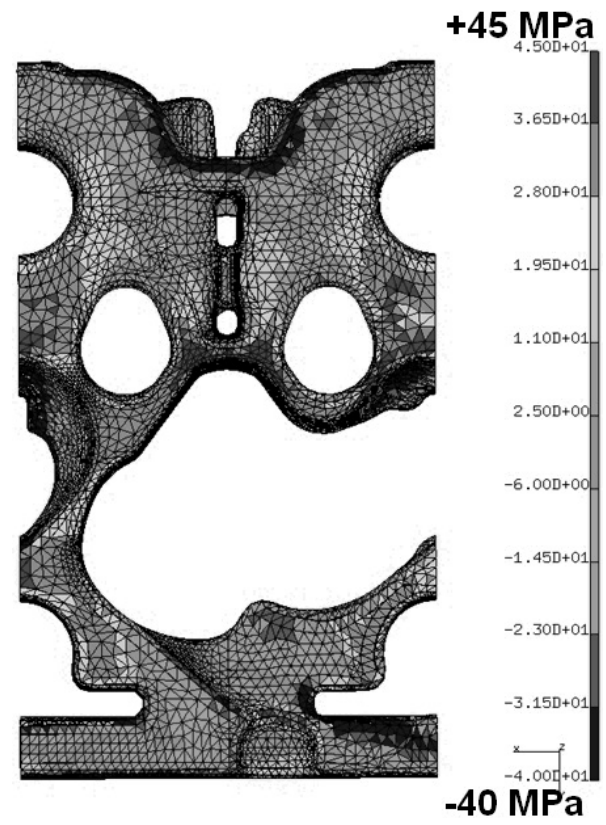

Fig. 6. Pression hydrostatique à la fin de la trempe.

Cette inversion s'explique par le fait que dans un premier temps l'extérieur se refroidit alors que l'intérieur est encore chaud et dans un second temps l'intérieur se refroidit à son tour avec un extérieur déjà froid et rigide.

Les résultats obtenus, figure 6 , à la fin de la trempe (et pareillement à la fin du revenu) montrent donc la présence de contraintes de traction dans la zone intérieure où la fissuration a été observée sur le prototype testé sur banc.

\section{Tenue en service de la culasse}

\subsection{Modélisation de la tenue en service}

Une fois les contraintes résiduelles déterminées, l'essai sur banc, utilisé pour valider la tenue en service de la zone froide de la culasse, est ensuite modélisé. Cet essai se compose d'un frettage des guides et des sièges de

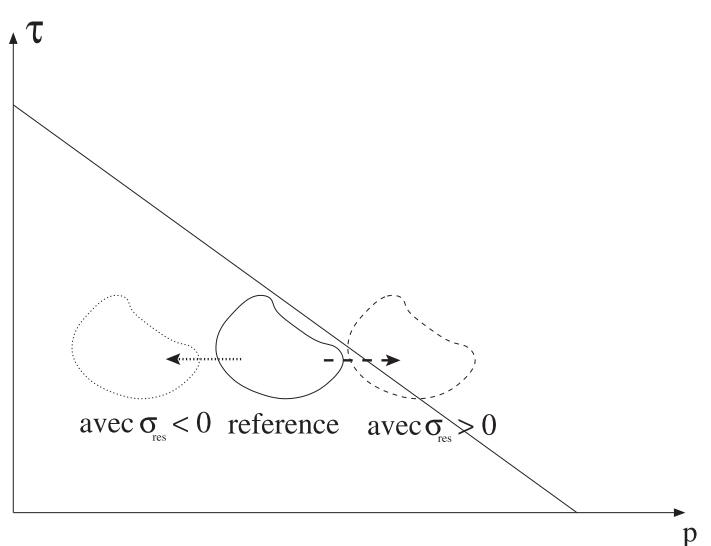

Fig. 7. Évolution d'un trajet de chargement dans le diagramme de Dang Van avec prise en compte des contraintes résiduelles.

soupape, d'un serrage de la culasse sur le bloc moteur, d'une montée en température et enfin d'un cyclage en pression (ce dernier correspondant aux cycles de combustion moteur). L'état résiduel (contraintes, déformations et variables internes) est réintroduit afin de tenir compte de l'état initial de la structure. L'état stabilisé (adaptation) de la structure est alors recherché afin de pouvoir appliquer un critère de tenue en fatigue.

\subsection{Influence des contraintes résiduelles sur la tenue en service}

Pour garantir la tenue en service d'une pièce travaillant dans le domaine de la fatigue polycyclique (i.e. à partir de $10^{6}$ cycles), le critère de Dang Van est utilisé chez PSA [7]. Celui-ci suppose qu'une structure qui s'adapte (i.e. une plastification peut apparaître lors des premiers cycles mais l'état asymptotique est lui purement élastique) à l'échelle mésoscopique ne fissurera pas. Il s'exprime sous la forme :

$$
\forall t \tau(t)+a p(t)<b
$$

$\tau$ et $p$ étant le cisaillement maximal à l'échelle mésoscopique et la pression hydrostatique.

Schématiquement, si le trajet de chargement reste en dessous de la droite matériau aucune rupture ne se produit alors que si le trajet franchit la droite il y a amorçage de fissure de fatigue.

L'ajout de contraintes résiduelles ne modifiant pas les valeurs du cisaillement mésoscopique $\tau$ mais faisant varier la pression hydrostatique $p$, la prise en compte du process revient à translater le trajet de chargement suivant $p$. Graphiquement (Fig. 7), dans le diagramme de Dang Van, le trajet de chargement est donc décalé vers la gauche dans le cas de contraintes résiduelles de compression (la tenue en fatigue de la pièce est améliorée) et inversement vers la droite dans le cas de contraintes résiduelles de traction (les contraintes résiduelles sont défavorables pour la tenue). 

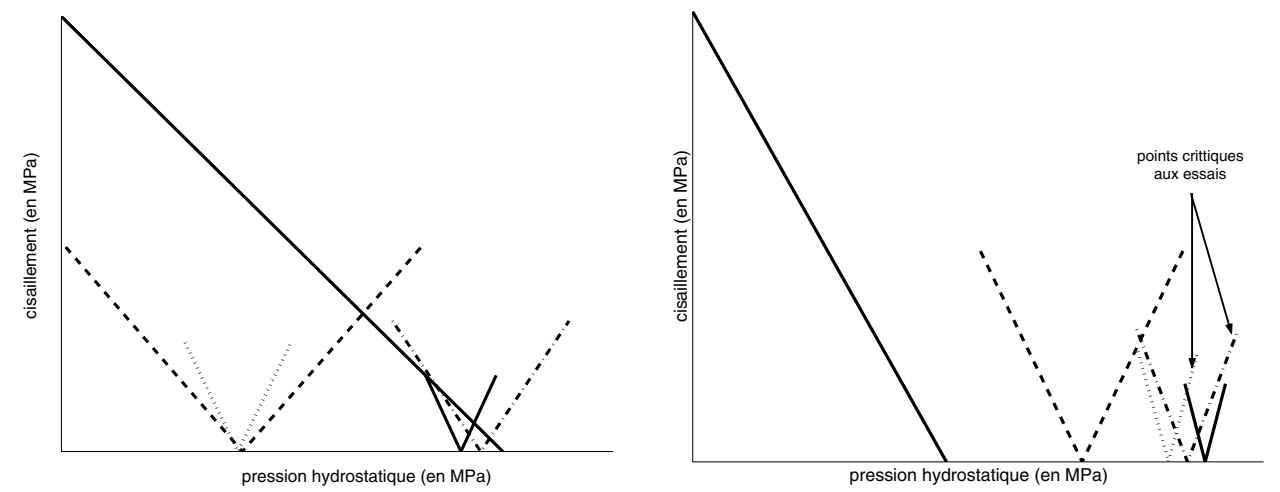

Fig. 8. Diagramme de Dang Van sans (gauche) ou avec (droite) prise en compte des contraintes résiduelles de process.

\subsection{Comparaison des résultats expérimentaux et numériques}

Les résultats numériques obtenus sont présentés dans la figure 8 . On observe que la prise en compte du process décale fortement les trajets de chargement des points critiques qui se trouvent désormais au-delà de la droite matériau. Le décalage s'effectue vers la droite pour tous les points de la figure puisqu'ils se situent tous dans une zone intérieure de la culasse et que les contraintes résiduelles y sont de traction. Cependant ce décalage n'est pas uniforme et, par rapport à un calcul où seule la tenue en service est modélisée, une inversion de l'ordre de certains points est observée. C'est cette translation, non uniforme, du trajet de chargement dans le diagramme de Dang Van qui justifie la modélisation du procédé de traitement thermique. Sa prise en compte permet alors d'obtenir une cohérence entre les résultats expérimentaux, où une fissuration a été observée sous le chargement sévérisé simulé, et les simulations numériques effectuées.

\subsection{Modification du procédé de trempe et influence sur la tenue à la fatigue polycyclique}

Les contraintes résiduelles obtenues à la fin du procédé de traitement thermique influent négativement sur la tenue à la fatigue polycyclique des culasses. Dans le cas où cette dernière ne peut plus être assurée une modification de la géométrie et/ou du process peut permettre de résoudre le problème. Les trempes à l'air sont un moyen permettant de fortement réduire le niveau des contraintes résiduelles.

Elles présentent l'avantage de refroidir plus homogènement la pièce en faisant circuler tout autour de celle-ci un courant d'air. Les écarts de température dans la pièce se trouvant alors fortement réduits, les contraintes résiduelles seront désormais plus faibles. Par rapport au cas de la trempe à l'eau, la tenue à la fatigue polycyclique s'en trouve donc améliorée. Ainsi, comme le montre la figure 9, la simulation d'une trempe à l'air avec un refroidissement en $210 \mathrm{~s}$, à comparer aux $40 \mathrm{~s}$ lors d'une trempe classique, permet de très fortement réduire les contraintes résiduelles et ainsi de grandement améliorer la tenue à la

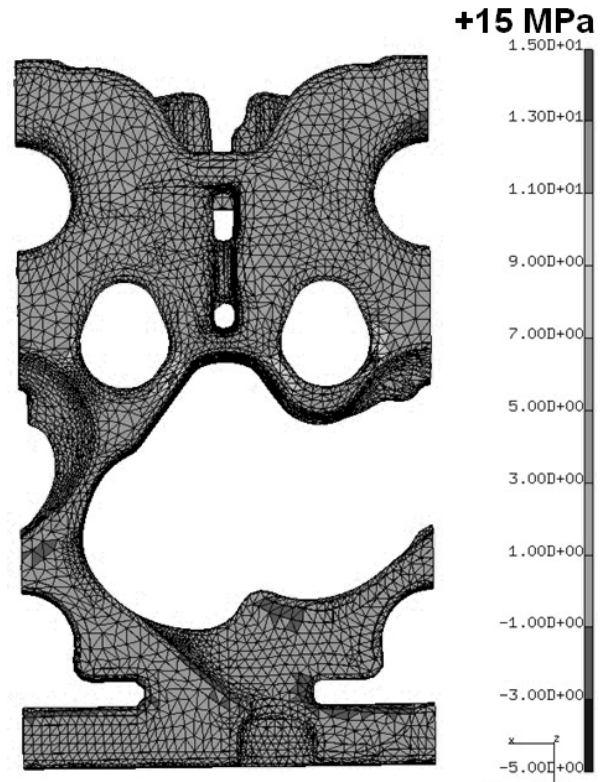

$-5 \mathrm{MPa}$

Fig. 9. Pression hydrostatique à la fin de la trempe à l'air (refroidissement de $210 \mathrm{~s}$ ).

fatigue polycyclique. Dans la zone à risque on passe ainsi d'une pression hydrostatique de $35 \mathrm{MPa}$ avec la trempe à l'eau à $5 \mathrm{MPa}$ avec le refroidissement à l'air. Après essais sur banc de culasses ayant subi ces deux traitements il a été observé que les culasses trempées à l'air avaient une bien meilleure endurance par rapport aux culasses trempées à l'eau.

Toutefois le refroidissement doit rester suffisamment rapide pour pouvoir figer la microstructure et ainsi obtenir, à la fin du traitement thermique, les propriétés mécaniques optimales. Un compromis est ainsi à déterminer sur la vitesse de refroidissement entre d'une part le niveau des contraintes résiduelles (qui augmente avec la vitesse de refroidissement) et d'autre part les caractéristiques mécaniques obtenues à la fin du traitement thermique (qui se trouvent améliorées avec une vitesse de trempe élevée).

D'autres évolutions du procédé comme la mise en solution étagée, les trempes à l'eau avec additifs, les trempes 
à l'huile etc. peuvent également être modélisées avec la méthodologie précédemment décrite et servir à évaluer l'intérêt d'éventuelles évolutions industrielles.

\section{Conclusions et perspectives}

La modélisation des échanges au cours du traitement thermique des culasses en aluminium et la simulation mécanique du process ont permis d'évaluer les contraintes résiduelles générées et ont montré la présence de contraintes de traction dans la zone critique observée aux essais. La prise en compte de ces contraintes résiduelles sévérise la criticité du trajet de chargement de service et est essentielle pour prédire la fissuration expérimentale.

Dans le cas où les contraintes résiduelles seraient trop pénalisantes il peut être possible d'envisager des évolutions du procédé de traitement thermique permettant de les diminuer. Les trempes à l'air, qui permettent d'obtenir, à la fin du process, un état de contraintes quasinul dans la zone à risque en sont un exemple.

Il faut cependant noter que la simulation du procédé de traitement thermique classique (trempe à l'eau-revenu) est extrêmement coûteuse en temps de calcul du fait de la taille des modèles (environ 800000 ddls), des lois de comportements non-linéaires utilisées et de la nécessité d'une discrétisation temporelle fine lors de la trempe. Pour contourner cette difficulté numérique, une méthode de calcul inspirée de la méthode à grand incrément de temps et de la méthode cyclique directe est en cours de développement [8].

\section{Références}

[1] P. Auburtin, N. Morin, Simulation thermomécanique $\mathrm{du}$ traitement thermique des culasses en aluminium, Mécanique \& Industries 4 (2003) 319-325

[2] H.M. Tensi, A. Stich, G.E. Totten, Applying fundamentals - fundamentals of quenching, INIST CNRS, 1999

[3] S.G. Chen, C. Weng, J. Lin, Inverse estimation of transient temperature distribution in the end quenching test, J. Mat. Proc. Tech. 86 (1999) 257-263

[4] D.E. Smith, Optimisation-based inverse heat transfert analysis for salt quenching of automotive components, Int. J. Vehicule Design 25(1/2) (2001) 23-39

[5] M. Ehlers, H. Müller, D. Löhe, Simulation of stresses and residual stresses due to immersion cooling of tempering steel, T. Ericsson, M. Odén, A. Andersson (ed.), The Fifth International Conference on Residual Stresses, Linköping, Sweden, 1997, vol. 1, pp. 400-405

[6] R. Jeschar, E. Specht, C. Köhler, Heat transfert during cooling of heated metallic objects with evaporating liquids, H.M. Tensi, B. Liscic, W. Luty (ed.), Theory and Technology of Quenching, Springer-Verlag, 1992, pp. $73-92$

[7] P. Ballard, K. Dang Van, A. Deperrois, Y.V. Papadopoulos, High cycle fatigue and a finite element analysis, Fatigue Fract. Engrg. Mater. Struct. 18(3) (1995) 397-411

[8] F. Comte, P. Burry, H. Maitournam, T.M.L. NguyenTajan, Sur une méthode directe de détermination de réponse transitoire : application à un problème thermomécanique, in Actes du septième colloque national en calcul de structures, Giens, 2005, à paraître

Retrouvez nos articles sur le site : www.edpsciences.org/meca 